\title{
Vestibular findings in patients with white matter disease
}

\author{
Nagwa Mohamed Hazzaa', Aya Yassin Ahmed², Amany Mohamed Abo El-Khair ${ }^{3}$ and Noha Ali Shafik ${ }^{1 *}$
}

\begin{abstract}
Background: Older people develop gait and balance dysfunction that is associated with the gradual onset of cerebral white matter disease. White matter disease is easily detected by neuroimaging, whereas small vessels are not; hence, the term small vessel disease is frequently used to describe the parenchyma lesions rather than the underlying small vessel alterations. This study was done to investigate the relationship between the presence and/or absence of white matter disease identified on magnetic resonance imaging with the vestibular findings in elderly patients with dizziness. The current study was conducted on a total number of 30 elderly patients complaining of dizziness and/or instability. The inclusion criteria are patients above 55 years old and a history of vertigo and/or instability. All the study groups were subjected to full neuro-otological history, hearing assessment, video-nystagmography test (VNG), assessment of the risk of fall by functional gait assessment test (FGA), and radiological assessment: magnetic resonance stroke protocol.

Results: FGA total scores were significantly lower in patients with white matter disease (0.047) specifically PVWM score 3 (0.04) and DWM score 3 (0.19). Abnormal VNG test results were significantly higher in patients with atherosclerotic changes (0.04). The most common VNG finding abnormality was positional nystagmus.

Conclusions: Normal VNG findings in elderly dizzy patients should be complemented with FGA test to assess the risk of falls. The presence of risk factors for white matter disease (WMD) as hypertension, diabetes mellitus, ischemic heart disease, hyperlipidemia, and migraine is an indication for requesting a further radiological assessment. The most frequent VNG abnormality in elderly patients with white matter disease is positional and positioning nystagmus, and a significant number of patients had positional nystagmus of the non-localizing criteria. FGA test scores are significantly related to the severity of white matter disease. The presence of atherosclerotic changes is significantly related to the presence of VNG test abnormality. It is important to ask the patient about the past history of migraine as it has an effect on VNG, FGA, and radiological findings.
\end{abstract}

Keywords: Elderly, White matter disease, Small vessel disease, Dizziness, FGA

\section{Background}

Age-related ischemic disease may manifest clinically with varying degrees and difficulties with mobility [1]. Dizziness and imbalance are some of the most common complaints in the elderly [2]. It is commonly associated with the gradual onset of age-related ischemic cerebral white

\footnotetext{
*Correspondence: nohashafik4@gmail.com

1 ENT Department, Faculty of Medicine, Ain Shams University, Cairo, Egypt Full list of author information is available at the end of the article
}

matter disease [3]. However, the mechanism through which it affects the gait is unknown $[4,5]$.

Several theories were proposed to explain the mechanism of gait disturbances in the elderly [6]. VNG as the gold standard tool for assessment of dizziness and vertigo in the elderly has a great role in the diagnosis of those patients; many VNG abnormalities particularly positioning and/or positional nystagmus were encountered in the VNG test in elderly patients [7]. 
Mankekar et al. [8] reported that central vestibular findings had a $50 \%$ positive predictive value for magnetic resonance imaging abnormalities.

To the best of the authors' knowledge, there are limited researches that examined the relation between agerelated ischemic changes as a radiological finding in MRI and MRA and the vestibular findings detected by VNG in elderly patients complaining of dizziness and/or imbalance. Therefore, the present study is designed to evaluate the vestibular findings using VNG in elderly patients and its relation to white matter changes detected by magnetic resonance imaging.

\section{Methods}

The current study was conducted on thirty elderly patients complaining of dizziness and/or instability who were selected from the audio-vestibular clinic of ElDemerdash Hospital, during the period from February 2018 to October 2018.The inclusion criteria are age group above 55 years old and positive history of vertigo and/or instability.

The exclusion criteria are patients diagnosed with known causes for dizziness as Meniere's disease, bilateral vestibulopathy, recent vestibular neuritis or labyrinthitis, history of head trauma or surgery, neurological diseases (intracranial space-occupying lesions, multiple sclerosis, parkinsonism and paresis), and patients with an otologic history of otorrhea or ear surgery.

\section{Equipment}

The following are the equipment:

- Sound treated room I.A.C model 1602.

- Two-channel audiometer interacustics, model AC40.

- Immitancemetery model otopront.

- Computerized video-nystagmography 2 channels (Micromedical Technologies, mobile eyes, spectrum 8.10).

- Magnetic resonance imaging stroke protocol (MRI and MRA).

- Functional gait assessment (FGA) tools, including stopwatch and two boxes; the height of each is $11 \mathrm{~cm}$.

\section{Method}

Each participant was subjected to the following:

- Full neuro-otological history

- Hearing assessment in the form of otological examination and pure tone audiometry: air conduction thresholds at $0.25,0.5,1,2,4$, and $8 \mathrm{KHz}$; bone conduction thresholds at $0.5,1,2$, ad $4 \mathrm{KHz}$; speech audiometry; and acoustic immittance testing
- Vestibular assessment

Video-nystagmography test (VNG), VNG test battery included the following tests:

- Spontaneous nystagmus

- Oculomotor tests: random saccade, smooth pursuit, optokinetic testing, and gaze test

- Positioning test (Dix-Hallpike, roll test)

- Positional nystagmus

- Caloric testing

The caloric test was done using the "alternate binaural bithermal" caloric method (automated open-loop water irrigation).

Output is using the average of peak slow-phase velocity response of the four irrigations, the software utilized Jongkees' formula to calculate the asymmetry between the ears [9].

The percentage of caloric weakness was considered to be significant at the level of $20 \%$ and more, while signs of uncompensation included the presence of spontaneous nystagmus, positional nystagmus, or significant directional preponderance of more than 25\% [10].

Videonystagmography required an average of $30-45$ min.

\section{Assessment of risk of fall by functional gait assessment test}

The functional gait assessment (FGA) is a standardized test for assessing postural stability during various walking tasks. The test is a modified version of the Dynamic Gait Index test which was developed [11]. Wrisley et al. [12] revised the DGI test to create the FGA test.

The FGA is a 10-item clinical gait test during which participants are asked to perform the following gait activities: walk at normal speeds, at fast speeds, and at slow speeds, with vertical and horizontal head turns, with eyes closed, over obstacles, in tandem, backward, and while ascending and descending stairs. The FGA is scored on a 4-level (0-3) ordinal scale; scores range from 0 to 30, with lower scores indicating greater impairment [13-15].

The FGA test was performed in all patients except 1 due to poor general condition. The instructions of the items were understood by the subjects of the study. The time needed to complete the testing was about 10 to 15 min.

\section{Radiological assessment}

Magnetic resonance stroke protocol is a group of magnetic resonance sequences put together to best approach brain ischemia: consists of T1, T2, T2/FLAIR, diffusion (DWI) images, and MR angiography (MRA) [16-20]. 
Fazekas classification [21] divides the white matter in periventricular (PVWM) and deep white matter (DWM) and is given a grade depending on the size and confluence of lesions on a scale range from 0 to 3 with the highest grade indicating more severe lesions.

\section{Statistical methods}

Data were coded and entered using the statistical package for the Social Sciences (SPSS) version 26 (IBM Corp., Armonk, NY, USA). Data was summarized using mean and standard deviation for quantitative variables and frequencies (number of cases) and relative frequencies (percentages) for categorical variables. Comparisons between the groups were done using unpaired $t$ test when comparing 2 groups and analysis of variance (ANOVA) with multiple comparisons post hoc test when comparing more than 2 groups [22]. For comparing categorical data, the chi-square $\left(\chi^{2}\right)$ test was performed. Exact test was used instead when the expected frequency is less than 5 [23]. Correlations between quantitative variables were done using the Pearson correlation coefficient [24]. P values less than 0.05 were considered statistically significant.

\section{Results}

Please see the results in Tables 1, 2, 3, 4, 5, 6, 7, 8, 9, 10, and 11 .

\section{Discussion}

In the present work, the authors are demonstrating the vestibular results of 30 dizzy patients in addition to their radiological findings. In 25 of them, no clear diagnosis could be reached after full history taking and VNG testing; 5 patients had BPPV either the only VNG finding (2 patients) or accompanied by other VNG findings (3patients) (Table 2).

All subjects who underwent radiological investigations had one or more abnormal findings, $78.6 \%$ had white matter disease (WMD), 71.4\% had atherosclerotic changes, and $78.5 \%$ had mixed lesions (Table 3). VNG showed abnormal test results in $60 \%$ (Table 2 ) and all had reduced FGA test scores (Table 5).

Table 2 shows that $60 \%$ of the study group had abnormal VNG findings in the form of peripheral, non-localizing, and mixed (central and peripheral) vestibular lesions. The number of abnormal patients $60 \%$ is lesser than the total number of VNG test findings due to the presence of multiple test findings in the same patient.

"Peripheral vestibular lesion based on the positioning (Dix-Hallpike), peripheral criteria of positional nystagmus as the only finding, and unilateral caloric weakness.

"Non-localizing vestibular lesion based on the presence of positional nystagmus as the only VNG finding with the criteria of positional nystagmus not clearly
Table 1 Distribution of patients according to VNG test findings

\begin{tabular}{|c|c|c|c|c|}
\hline \multirow[t]{2}{*}{ VNG findings } & \multicolumn{2}{|l|}{ No abnormality } & \multicolumn{2}{|l|}{ Abnormal } \\
\hline & No of patients & $\%$ & No of patients & $\%$ \\
\hline $\begin{array}{l}\text { Spontaneous nystag- } \\
\text { mus }\end{array}$ & 26 & 86.7 & 4 & 13.3 \\
\hline Gaze test & 30 & 100 & 0 & 0 \\
\hline Optokinetic & 30 & 100 & 0 & 0 \\
\hline Pursuit & 30 & 100 & 0 & 0 \\
\hline Saccade & 30 & 100 & 0 & 0 \\
\hline Positioning & 25 & 83.3 & 5 & 16.7 \\
\hline${ }^{b}$ Positional & 17 & 56.7 & 13 & 43.3 \\
\hline $\begin{array}{l}\text { 'Unilateral caloric weak- } \\
\text { ness }\end{array}$ & 26 & 86.7 & 4 & 13.3 \\
\hline
\end{tabular}

N.B.: No isolated central vestibular deficit was detected in the study group. Some patients had multiple VNG abnormalities

a There was an abnormality in positioning test in 5 patients; 3 of them had in addition to the positioning nystagmus either spontaneous or positional nystagmus (of mixed peripheral and central criteria of vestibular lesion) as follows: vertical nystagmus, one of them diminished while others abolished or diminished with fixation, with a minimal subjective sense of dizziness

${ }^{b}$ The other patients with positional nystagmus were either peripheral with the following criteria: horizontal nystagmus, directional fixed, abolished with fixation and with a subjective sense of dizziness, or mixed peripheral and central criteria of vestibular lesion as follows: vertical nystagmus, abolished or diminished with fixation with a minimal subjective sense of dizziness

c $13.3 \%$ of the study group had unilateral caloric weakness; 2 of them were uncompensated as accompanied with spontaneous or positional nystagmus and the other 2 were compensated with unilateral caloric weakness as the only VNG finding

matching a peripheral or central site of lesion (vertical nystagmus, abolished or diminished with fixation with a minimal subjective sense of dizziness).

***:Mixed criteria of vestibular lesion based on the presence of multiple VNG finding including BPPV together with spontaneous nystagmus (2patients) and/ or positional nystagmus of mixed criteria (peripheral and central) as mentioned above. One of the patients had unilateral caloric weakness in addition to the positioning, positional and spontaneous nystagmus.

The most common risk factors for WMD in the present study were hypertension (53.3\%) and diabetes mellitus (43.3\%). Other risk factors included hyperlipidemia $(30 \%)$, ischemic heart disease (23.3\%), and migraine (16.7\%), and $46.7 \%$ of the study group had more than one risk factor (Table 4). Arterial hypertension was found to be the most common risk factor of the dizziness associated with white matter disease in elderly patients with a percentage of $81 \%$. Cardiovascular factors also could have a pathogenic role in WMD as well as in vestibular disorders and in aggravating symptoms of both diseases [25].

Regarding FGA test scores, there were tasks that were expected to be more difficult for people with vestibular disorders and they were ambulating backwards, gait with 
Table 2 Distribution of the study group according to the site of the lesion based on the VNG test

\begin{tabular}{|c|c|c|c|c|}
\hline \multicolumn{3}{|l|}{ VNG test results } & \multirow{2}{*}{$\begin{array}{c}\text { No. } \\
12\end{array}$} & \multirow{2}{*}{$\begin{array}{l}\% \\
40\end{array}$} \\
\hline Normal VNG study & & & & \\
\hline \multirow[t]{5}{*}{ Abnormal VNG } & ${ }^{\text {aPeripheral vestibular lesion }}$ & BPPV & 2 & 6.7 \\
\hline & & Positional (peripheral) & 6 & 20 \\
\hline & & Unilateral caloric weakness & 3 & 10 \\
\hline & ${ }^{\mathrm{b}}$ Non-localizing positional criteria & & 4 & 13.3 \\
\hline & ${ }^{c}$ Mixed vestibular lesion & & 3 & 10 \\
\hline \multicolumn{3}{|c|}{ Total patient in the study group } & 30 & 100 \\
\hline
\end{tabular}

${ }^{a}$ Peripheral vestibular lesion based on the positioning (Dix-Hallpike), peripheral criteria of positional nystagmus as the only finding, and unilateral caloric weakness

${ }^{b}$ Non-localizing vestibular lesion based on the presence of positional nystagmus as the only VNG finding with the criteria of positional nystagmus not clearly matching a peripheral or central site of lesion (vertical nystagmus, abolished or diminished with fixation with a minimal subjective sense of dizziness)

${ }^{c}$ Mixed criteria of the vestibular lesion based on the presence of multiple VNG findings including BPPV together with spontaneous nystagmus ( 2 patients) and/or positional nystagmus of mixed criteria (peripheral and central) as mentioned above. One of the patients had unilateral caloric weakness in addition to the positioning, positional, and spontaneous nystagmus

Table 3 Distribution of patients according to different radiological findings. N.B: Two patients were excluded from 30 patients due to contraindication of MRI due to metallic heart prosthesis and metallic clips, so the total radiological investigated patients were 28

\begin{tabular}{|c|c|c|c|c|c|c|}
\hline & \multicolumn{2}{|c|}{ Present } & \multicolumn{2}{|c|}{ Absent } & \multicolumn{2}{|c|}{ Total } \\
\hline & No. & $\%$ & No. & $\%$ & No. & $\%$ \\
\hline Involution brain changes & 15 & 53.6 & 13 & 46.4 & 28 & 100 \\
\hline White matter disease & 22 & 78.6 & 6 & 21.4 & 28 & 100 \\
\hline Old multi-lacunar infarction & 12 & 42.9 & 16 & 57.1 & 28 & 100 \\
\hline Atherosclerotic changes & 20 & 71.4 & 8 & 28.6 & 28 & 100 \\
\hline Mixed lesion ${ }^{\mathrm{a}}$ & 22 & 78.57 & 6 & 21.42 & 28 & 100 \\
\hline
\end{tabular}

${ }^{\text {a }}$ Mixed lesion based on the presence of more than one radiological finding in the same patient. No single patient was normal in the radiological assessment

Table 4 Distribution of patients according to known medical history

\begin{tabular}{llllll}
\hline Risk factors $^{\text {a }}$ & \multicolumn{2}{l}{ Present } & & \multicolumn{2}{l}{ Absent } \\
\cline { 2 - 3 } & No. & $\%$ & & No. & \% \\
\hline Diabetes & 13 & 43.3 & & 17 & 56.7 \\
Hypertension & 16 & 53.3 & & 14 & 46.7 \\
Ischemic heart disease & 7 & 23.3 & & 23 & 76.7 \\
Hyperlipidaemia & 9 & 30.0 & & 21 & 70.0 \\
Migraine & 5 & 16.7 & & 25 & 83.3 \\
bultiple risk factor & 14 & 46.7 & & 16 & 53.3 \\
\hline
\end{tabular}

The most common risk factors were hypertension (53.3\%) and diabetes (43.3\%), and $46.7 \%$ of the study group had more than one risk factor

${ }^{\text {a }}$ All patients had at least one risk factor for WMD

${ }^{\mathrm{b}}$ In some patients, there were multiple risk factors in the same patient

eyes closed, gait with a narrow base of support, and tasks involving head turns $[26,27]$. Our results showed a high percentage of patients having difficulty in performing those tasks (Table 5) that could be due to the large percentage of patients having a vestibular lesion in the study group.
In subjects with WMD and SVD, gait speed was found to be significantly slower, and it was assessed by the $6-\mathrm{m}$ walk test [28]. The same findings were evident in our study group although using simple office tests as all the patients performed the FGA tasks have abnormal gait level surface task (task no. 1) regarding the speed of the task rather than deviation (Table 5).

The value of the present work can be looked for from two prospectives; the first is the normal VNG test results in $40 \%$ of patients. In cases of elderly "or adults above the age of 55 years," testing should not stop at this stage. The present study showed that a simple office test as FGA is mandatory to be conducted and is very beneficial. All subjects in this group (including the $40 \%$ normal VNG) (Table 2) had decreased FGA scores (Table 5) and one or more radiological abnormalities as detected by MRI and/ or MRA (Table 3).

Furthermore, 50\% of normal VNG subjects had atherosclerotic changes as detected by MRA compared to $87.5 \%$ of the abnormal VNG group (Table 6). The chi-square test results showed a statistically significant difference $(P$ value $=0.044$ ). Elderly dizzy patients with normal VNG findings who have more risk factors for WMD as diabetes 
Table 5 Number and percentage of patients of the study group according to FGA different grades

\begin{tabular}{|c|c|c|c|c|c|c|c|c|c|c|}
\hline \multirow[t]{2}{*}{ Tasks } & \multicolumn{2}{|c|}{ Normal } & \multicolumn{2}{|c|}{ Mild } & \multicolumn{2}{|c|}{ Moderate } & \multicolumn{2}{|c|}{ Severe } & \multicolumn{2}{|c|}{ Total patients } \\
\hline & $N$ & $\%$ & $N$ & $\%$ & $N$ & $\%$ & $N$ & $\%$ & 29 & 100 \\
\hline 1. Gait level surface & 0 & 0 & 4 & 13.8 & 21 & 72.4 & 4 & 13.8 & 29 & 100 \\
\hline 2. Change in gait speed & 19 & 65.5 & 7 & 24.1 & 3 & 10.3 & 0 & 0 & 29 & 100 \\
\hline 3. Gait with horizontal head turns & 4 & 13.8 & 21 & 72.4 & 4 & 13.8 & 0 & 0 & 29 & 100 \\
\hline 4. Gait with vertical head turns & 13 & 44.8 & 16 & 55.2 & 0 & 0 & 0 & 0 & 29 & 100 \\
\hline 5. Gait and pivot turns & 23 & 79.3 & 5 & 17.2 & 1 & 3.4 & 0 & 0 & 29 & 100 \\
\hline 6. Step over an obstacle & 25 & 86.2 & 2 & 6.9 & 2 & 6.9 & 0 & 0 & 29 & 100 \\
\hline 7. Gait with a narrow base of support & 9 & 31 & 4 & 13.8 & 10 & 34.5 & 6 & 20.7 & 29 & 100 \\
\hline 8. Gait with eyes closed & 1 & 3.4 & 3 & 10.3 & 18 & 62.1 & 7 & 24.1 & 29 & 100 \\
\hline 9. Ambulating backwards & 10 & 34.5 & 16 & 55.2 & 2 & 6.9 & 1 & 3.4 & 29 & 100 \\
\hline 10. Steps & 15 & 51.7 & 4 & 13.8 & 10 & 34.5 & 0 & 0 & 29 & 100 \\
\hline Total score & 0 & 0 & 17 & 58.6 & 12 & 41.4 & 0 & 0 & 29 & 100 \\
\hline
\end{tabular}

All of the patients had mild and moderate degrees in FGA

*The mean and SD of the patients in the study group is $20.4 \pm 4.1$ for the total patients who performed FGA test in the study group, while it ranges from $23.2 \pm 1.9$ in the patients with mild FGA impairment to $16.4 \pm 2.7$ in the patients with moderate FGA impairment

Table 6 The chi-square test between VNG test results and different radiological findings

\begin{tabular}{|c|c|c|c|c|c|c|}
\hline & & \multicolumn{4}{|c|}{ VNG results } & \multirow[t]{3}{*}{$P$ value } \\
\hline & & \multicolumn{2}{|c|}{ Normal } & \multicolumn{2}{|c|}{ Abnormal } & \\
\hline & & No. & $\%$ & No. & $\%$ & \\
\hline \multirow[t]{2}{*}{ Atherosclerotic changes } & Yes & 6 & 50.0 & 14 & 87.5 & 0.044 \\
\hline & No & 6 & 50.0 & 2 & 12.5 & \\
\hline \multirow[t]{2}{*}{ Old lacunar inf. } & Yes & 4 & 33.3 & 8 & 50.0 & 0.378 \\
\hline & No & 8 & 66.7 & 8 & 50.0 & \\
\hline \multirow[t]{2}{*}{ WMD } & Yes & 9 & 75.0 & 13 & 81.3 & 1 \\
\hline & No & 3 & 25.0 & 3 & 18.8 & \\
\hline \multirow[t]{2}{*}{ Involution brain changes } & Yes & 7 & 58.3 & 8 & 50.0 & 0.662 \\
\hline & No & 5 & 41.7 & 8 & 50.0 & \\
\hline
\end{tabular}

The presence of atherosclerotic changes as a finding detected by MRA was statistically significantly higher in patients having abnormal VNG test findings $(P$ value $=$ 0.04)

Table 7 Distribution of the patients according to Fazekas scoring

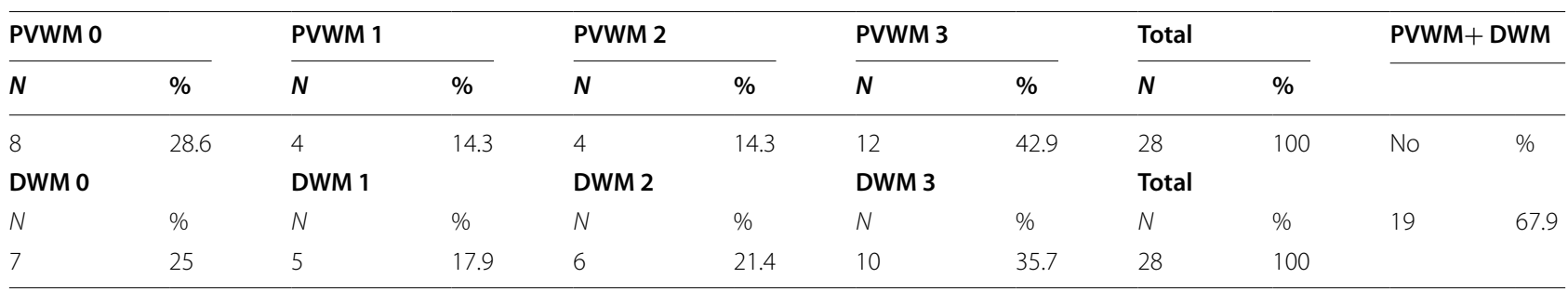

The most common Fazekas scorings in the study group are grade 3 WMD (PVWM and DWM) and combined lesion of both PVWM and DWM

or hypertension should be subjected to FGA and radiological studies to receive appropriate medical treatment if needed.
Another interesting finding in the present work is the normal occulomotor test results in all subjects (Table 1). Contrary to the present study, another study found that there was a positive predictive value of 
Table 8 Unpaired $t$ test between FGA total score and radiological findings as detected by magnetic resonance imaging (MRI and MRA)

\begin{tabular}{lllll}
\hline & & \multicolumn{2}{l}{ FGA total score } & P value \\
\cline { 2 - 4 } & & Mean & $\begin{array}{c}\text { Standard } \\
\text { deviation }\end{array}$ & \\
\hline Atherosclerotic changes & Yes & 20.58 & 4.65 & 0.923 \\
& No & 20.75 & 2.38 & \\
Old lacunar inf. & Yes & 20.75 & 3.86 & 0.894 \\
\multirow{2}{*}{ WMD } & No & 20.53 & 4.34 & \\
\multirow{2}{*}{ Involution brain changes } & Yes & 19.81 & 3.98 & 0.047 \\
& No & 23.50 & 3.08 & \\
& No & 21.00 & 4.57 & 0.632 \\
\hline
\end{tabular}

Patients with WMD as a radiological finding detected by MRI had lower FGA total scores $(P$ value $=0.047)$

Table 9 Unpaired $t$ test between PVWM 0 and PVWM 3 with the FGA total scores

\begin{tabular}{llllllll}
\hline & \multicolumn{2}{l}{ PVWML 0 } & & \multicolumn{2}{l}{ PVWML 3 } & P value \\
\cline { 2 - 3 } & Mean & SD & & Mean & SD & \\
\hline FGA total score & 22.88 & 3.04 & & 18.73 & 4.56 & 0.040 \\
\hline
\end{tabular}

Patients with PVWM 3 had lower FGA total scores $(P$ value $=0.04)$ lesion, and abnormal occulomotor abnormalities can be expected [8].

Moreover, occulomotor abnormalities are often accompanied by CNS diseases with symptoms such as blurred vision, oscillopsias, double vision, or staggering [29]. Such conditions were among the exclusion criteria in the present study.

Concerning subjects with abnormal VNG findings (60\% of the study group), they demonstrate either peripheral site of lesion $(36.7 \%)$, combined site of lesion $(10 \%)$, or the site could not be otherwise specified (26.3\%) as shown in Table 2. This agreed with other studies who found that there was a higher prevalence of pathological vestibular signs of a peripheral type in patients with WMD [30, 31]. Also, other researchers found that BPPV was found to be the main cause of dizziness in $26 \%$ of patients with WMD (Fazekas score 2-3) [31].

In the present study, the most frequent VNG abnormality was the presence of positional nystagmus in $43.3 \%$ of patients (Table 1), some of peripheral and other with nonlocalizing criteria. In the present authors' experience, the presence of positional nystagmus of non-localizing character as "the only abnormality in VNG" is almost always related to the presence of age-related ischemic changes [7]. In fact, peripheral vestibular involvement or CNS involvement may provide similar findings during testing. The delineation between the peripheral and central sites of the lesion within the vestibular system based on the

Table 10 ANOVA test between FGA total score and Fazekas scores (DWM)

\begin{tabular}{|c|c|c|c|c|c|c|c|c|c|}
\hline & \multicolumn{8}{|l|}{ DWM } & \multirow[t]{3}{*}{$P$ value } \\
\hline & \multicolumn{2}{|c|}{ DWM 0} & \multicolumn{2}{|c|}{ DWM 1} & \multicolumn{2}{|c|}{ DWM 2} & \multicolumn{2}{|c|}{ DWM 3} & \\
\hline & Mean & SD & Mean & SD & Mean & SD & Mean & SD & \\
\hline FGA total score & 23.29 & 2.87 & 22.60 & 1.95 & 20.33 & 3.67 & 17.67 & 4.30 & 0.019 \\
\hline
\end{tabular}

Table 11 Post hoc pair-wise comparisons between the FGA total scores and different grades of DWM

\begin{tabular}{lllll}
\hline & DWM 0 & DWM 1 & DWM 2 & DWM 3 \\
\hline DWM 0 & & 0.987 & 0.442 & 0.020 \\
DWM 1 & 0.987 & & 0.709 & 0.081 \\
DWM 2 & 0.442 & 0.709 & & 0.482 \\
DWM 3 & 0.020 & 0.081 & 0.482 &
\end{tabular}

FGA total scores were statistically significantly higher in patients with DWM 0 than in patients with DWM $3(23.29 \pm 2.87$ vs $17.67 \pm 4.3, P$ value $=0.02)$

abnormal occulomotor test findings in relation to magnetic resonance imaging abnormalities. Compared to the present work, their group included cortical and cerebellar atrophy subjects' accordingly central vestibular criteria of positional nystagmus alone should be avoided. It was reported that positional tests could evaluate the state of cerebral perfusion contralateral to the direction of head movement [32]. In agreement with the present results, it was reported that lack of fixation suppression of positional nystagmus supports CNS involvement, but it is also not uncommon for some suppression to occur. Both age-related CNS changes and migraine may be the cause of positional nystagmus. They also reported an imperative need for correlation with patient history and symptoms, in addition to the results of VNG and other diagnostic evaluations [33].

In the present study, 5 patients had BPPV, 2 of them had the disease diagnosed by positioning Dix-Hallpike test alone while the rest of this group (3 subjects) had in addition positional nystagmus of non-localizing 
character (Tables 1 and 2). The causes of BPPV are primarily believed to be mechanical $[34,35]$. But the BPPV recurrence rate is approximately $12-20 \%$ [36, 37]. Approximately $2.4 \%$ of the population will at some point in their life be affected by BPPV, among individuals who live into their 80 s, the rate is as high as $10 \%$ with the onset typically being between 50 and 70 years [38]. Accordingly, conditions that are comorbid with BPPV have been rigorously investigated in the literature.

It is reported that BPPV patients with hypertension and hyperlipidemia are more likely to experience recurrence [39]. Moreover, age, migraine, hypertension, hyperlipidemia, and stroke were found to be independently associated with BPPV [40]. In addition, it was found that the prevalence of hypertension, diabetes mellitus, atrial fibrillation, coronary artery disease, and hyperlipidemia is higher among patients with BPPV than among those without BPPV [41].

The clinical implication of these findings in patients with BPPV is remarkable. The full VNG test battery is recommended in persistent dizziness after a successful repositioning maneuver. The underlying cause of such dizziness may be due to the presence of combined condition as WMD. In the latter case, neurological consultation, radiological assessment, and medical treatment are warranted.

In the present study, there were 5 patients with a past history of migraine (Table 4). Among those patients, there were 3 patients with abnormal VNG test findings in the form of positional nystagmus of peripheral criteria, and 2 patients showed normal VNG test results. Furthermore, all the patients had an abnormality in the radiological assessment. In a systemic review and meta-analysis, it was found that migraine may be a risk factor for structural changes in the brain including WMLs, lacunar infarction, and brain involutional changes [42].

In the present study, $13.3 \%$ of the study group have unilateral caloric weakness $50 \%$ of them were compensated, and the other $50 \%$ were uncompensated (Tables 1 and 2). Regarding the compensated patients, it was found that the compensation process that follows vestibular loss may be the key to the structural reorganization of white matter tracts involved in balance maintenance [43]. As the process of compensation is accompanied by structural reorganization of white matter disease so patients with uncompensated unilateral caloric weakness may suffer from WMD in this stage as there are more burdens on the central vestibular pathway. Moreover, regarding the uncompensated patients, as the atherosclerotic changes and small vessel disease result in ischemia of the affected structure, this may delay the process of central compensation that will result in more burdens on the central vestibular system.
This agreed with another study that found a significant canal paresis in $(38.7 \%)$ of SVD and the dizziness was likely due to insufficient central compensation after a previous vestibular loss [30].

The most common radiological findings among the study group were white matter disease (WMD) (78.6\%) and atherosclerotic changes (71.4\%) (Table 3). Periventricular white matter Fazekas score 3 (PVWM 3) was found in $42.9 \%$, and deep white matter Fazekas score 3 (DWM 3) was found in $35.7 \%$ reflecting the degree of severity of WMD in the dizzy patients. Combined lesions (both PVWM and DWM) were also very prevalent $(67.9 \%)$ regardless of severity (Table 7).

Conducting statistical analysis of the present data showed that the presence of WMD (PVWM 3 and DWM 3) detected by MRI was associated with lower FGA total scores (Tables 8, 9, 10, and 11). Moreover, the presence of atherosclerotic changes detected by MRA was associated with a higher percentage of VNG abnormalities $(p=0.04)$ (Table 6).

Gait deterioration was found to be associated with white matter atrophy and was independent of age, gender, height, and follow-up duration [44]. It is reported also that the presence of WMD seems to be the major driving force for gait impairment in elderly subjects [26]. The cause of this correlation between dizzinessinduced gait abnormality and WMD could be the disruption of neuronal networks that mediate higher vestibular cortical function [25]. Also, a systematic review indicated that WMD are common in older people and are significantly associated with impaired balance, gait, mobility, and falls. However, in many studies, these findings were only evident in people who have the most severe degree of WMD [45].

Moreover, SVD is a dynamic and highly variable disease process with progression and in some regression of SVD $[46,47]$. In a recent study, reduction in blood pressure was associated with a reduction in WMD volume [47]. This suggests that better blood pressure control could attenuate or even reverse the WMD growth and consequently could lead to better gait function over time.

Age-related ischemic changes as a disease prevalent among older adults present with various degrees of dizziness and gait impairment. Dizzy patients with no clear etiology above the age of 55 years old have signs of white matter disease with variable degrees of severity and different radiological presentations.

The present results suggest that elderly patients with dizziness, without a clear diagnosis and despite clinical and vestibular assessment, should undergo brain imaging to assess the level of WMD and possible comorbid condition as presence of atherosclerotic changes. 


\section{Conclusion}

- The presence of risk factors of white matter disease (WMD) such as hypertension, diabetes mellitus, ischemic heart disease, hyperlipidemia, and migraine is a medical indication for requesting further radiological assessment in dizzy patients.

- The most frequent VNG abnormality in elderly patients with white matter disease is positional and positioning nystagmus, and a significant number of patients had positional nystagmus of non-localizing criteria.

- FGA test scores are significantly related to the severity of white matter disease.

- The presence of atherosclerotic changes is significantly related to the presence of VNG test abnormality.

- It is important to ask the patient about the past history of migraine as it has a reflection on VNG, FGA, and radiological findings.

\section{Acknowledgements}

Not applicable

\section{Authors' contributions}

$\mathrm{NH}$ contributed to the idea, funding, supervision, and analyses of the results. AY contributed to the analysis, revision, and funding of the research. NS contributed to the supervision and funding and participated in working cases and analysis of the results. AA contributes to working cases and writing. All authors have read and approved the final manuscript.

\section{Funding}

No available funding

\section{Availability of data and materials}

Data and materials were available. The datasets used and/or analyzed during the current study are available from the corresponding author on reasonable request.

\section{Declarations}

\section{Ethics approval and consent to participate}

The protocol was ethically approved by the ENT Department Board, the Research Ethical Committee, Faculty of Medicine, Ain Shams University. Reference number is not available. Informed verbal consent was taken from the participants.

\section{Consent for publication}

Not applicable

\section{Competing interests}

The authors declare that they have no competing interests.

\section{Author details}

${ }^{1}$ ENT Department, Faculty of Medicine, Ain Shams University, Cairo, Egypt.

${ }^{2}$ Radiology Department, Ain Shams University, Cairo, Egypt. ${ }^{3}$ ENT Department, Ahmed Maher Teaching, Cairo, Egypt.

Received: 9 July 2021 Accepted: 4 February 2022

Published online: 21 February 2022

\section{References}

1. Sorond FA, Gorelick PB (2019) Brain white matter: a substrate for resilience and a substance for subcortical small vessel disease. Brain Sci 9(8)

2. Iwasaki S, Yamasoba T (2015) Dizziness and imbalance in the elderly: agerelated decline in the vestibular system. Aging and Disease; International Society on Aging and Disease

3. Whitman GT, Tang T, Lin A, Baloh RW (2001) A prospective study of cerebral white matter abnormalities in older people with gait dysfunction. Neurology 57(6):990-994

4. De Laat KF, Tuladhar AM, Van Norden AGW, Norris DG, Zwiers MP, De Leeuw FE (2011) Loss of white matter integrity is associated with gait disorders in cerebral small vessel disease. Brain 134(1):73-83

5. Rosario B, Rosso A, Aizenstein H, Harris T, Newman A, Satterfield S, Studenski S, Yaffe K, Rosano C (2016) Cerebral white matter and slow gait: contribution of hyperintensities and normal-appearing parenchyma. J Gerontol 71(7):968-973

6. Kaski D, Rust HM, Ibitoye R, Arshad Q, Allum JHJ, Bronstein AM (2019) Theoretical framework for "unexplained" dizziness in the elderly: the role of small vessel disease. Progress Brain Res 248:225-240 Elsevier B.V

7. Hazzaa N (2018) VNG findings in patients with age related ischemic changes. Personal Communication

8. Mankekar G, Jeha G, Erbele I, Klumpp M, Sevy A, Mehta R, Arriaga MA (2019) Do central vestibular findings predict abnormal findings on magnetic resonance imaging? J Laryngology Otol 133(7):554-559

9. Stockwell CW (1987) Directional preponderance. ENG report. ICS medical

10. Jongkees LBW (1948) Value of the caloric test of the labyrinth. Arch Otolaryngol 48:402-401

11. Shumway-Cook A, Woollacott MH (1995) Assessment of postural disorders. in Motor control: theory and practical applications. Williams \& Wilkins, Michigan, p 475

12. Wrisley DM, Pavlou M (2005) Physical therapy for balance disorders. Neurol Clin

13. Wrisley DM, Marchetti GF, Kuharsky DK, Whitney SL (2004) Reliability, internal consistency, and validity of data obtained with the functional gait assessment. Physical Ther

14. Wrisley DM, Kumar NA (2010) Functional gait assessment: concurrent, discriminative, and predictive validity in community-dwelling older adults. Phys Ther 90(5):761-773

15. Tawfik S, Hazzaa N, El Mowafy S (2010) Assessment of risk of fall in patients with peripheral vestibular disorders. Unpublished thesis

16. Schellinger PD, Jansen O, Fiebach JB, Hacke W, Sartor K (1999) A standardized MRI stroke protocol: comparison with CT in hyperacute intracerebral hemorrhage. Stroke 30(4):765-768

17. Wintermark M, Albers G, Alexandrov A, Alger J, Bammer R, Baron J, Davis S, Demaerschalk B, Derdeyn C, Donnan G, Eastwood J, Fiebach J, Fisher M, Furie K, Goldmakher G, Hacke W, Kidwell C, Kloska S, Köhrmann M, Koroshetz W, Lee T, Lees K, Lev M, Liebeskind D, Ostergaard L, Powers W, Provenzale J, Schellinger P, Silbergleit R, Sorensen A, Wardlaw J, Wu O, Warach S (2008) Acute stroke imaging research roadmap. Am J Neuroradiol 39(5):1621-1628 ISSN 0039-2499

18. Allen LM, Hasso AN, Handwerker J, Farid H (2012) Sequence-specific MR imaging findings that are useful in dating ischemic stroke. Radiographics 32(5):1285-1297

19. Srinivasan A, Goyal M, Al Azri F, Lum C (2006) State-of-the-art imaging of acute stroke. Radiographics. 26(supplement):1. https://doi.org/10.1148/ rg.26si065501

20. Kim AC, Vu D, Gilberto González R, Schaefer PW (2006) Conventional MRI and MR angiography of stroke. In: Acute Ischemic Stroke: Imaging and Intervention. Springer, Berlin Heidelberg, pp 123-144

21. Fazekas F, Chawluk JB, Alavi A, Hurtig HI, Zimmerman RA (1987) MR signal abnormalities at 1.5 T in Alzheimer's dementia and normal aging. Am J Roentgenol 149(2):351-356

22. Chan YH (2003a) Biostatistics102: quantitative data - parametric \& nonparametric tests. Singapore Med J. 44(8):391-396

23. Chan YH (2003b) Biostatistics 103: qualitative data -tests of independence. Singapore Med J. 44(10):498-503

24. Chan YH (2003c) Biostatistics 104: correlational analysis. Singapore Med J. 44(12):614-619

25. Gamba P, Pavia M (2016) White matter lesions and vascular vertigo: clinical correlation and findings on cranial magnetic resonance imaging. Eur Rev Med Pharmacol Sci 20(13):2786-2791 
26. Wrisley DM, Marchetti GF, Kuharsky DK, Whitney SL (2004) Reliability, internal consistency, and validity of data obtained with the functional gait assessment. Phys Ther. 84(10):906-918 PMID: 15449976

27. Chiu YP, Fritz SL, Light KE, Velozo CA (2006) Use of item response analysis to investigate measurement properties and clinical validity of data for the Dynamic Gait Index. Physical Ther 86(6):778-787

28. Pinter D, Ritchie S, Doubal F, Gattringer T, Morris Z, Bastin M, Hernández M, Royle N, Corley J, Muñoz Maniega S, Pattie A, Dickie D, Staals J, Gow A, Starr J, Deary I, Enzinger C, Fazekas F, Wardlaw J (2018) Impact of small vessel disease in the brain on gait and balance. Scientific Reports Aging 10(1):144-153

29. Strupp M, Hüfner K, Sandmann R, Zwergal A, Dieterich M (2011) Jahn K \& Brandt T (2011) Central oculomotor disturbances and nystagmus. Deutsches Aerzteblatt 108(12):197-204

30. Cerchiai N, Mancuso M, Navari E, Giannini N, Casani AP (2017) Aging with cerebral small vessel disease and dizziness: the importance of undiagnosed peripheral vestibular disorders. Front Neurol:8

31. Ahmad H, Cerchiai N, Mancuso M, Casani AP, Bronstein AM (2015) Are white matter abnormalities associated with "unexplained dizziness"? J Neurol Sci 358(1-2):428-431

32. Thomas L, Treleaven J (2020) Should we abandon positional testing for vertebrobasilar insufficiency? Musculoskelet Sci Pract 46

33. Shephard N, Telian S (1996) Electronystagmography evaluation. In: Practical management of the balance disorder patient. Singular, San Diego, pp 51-84

34. Baloh RW, Sloane PD, Honrubia V (1989) Quantitative vestibular function testing in elderly patients with dizziness. Ear, Nose Throat J 68(12):935-939

35. Neuhauser H, Leopold M, Von Brevern M, Arnold G, Lempert T (2001) The interrelations of migraine, vertigo, and migrainous vertigo. Neurology 56(4):436-441

36. Steenerson RL, Cronin GW, Marbach P (2005) Effectiveness of treatment techniques in 923 cases of benign paroxysmal positional vertigo. Laryngoscope 115(2):226-231. https://doi.org/10.1097/01.mlg.0000154723. 55044.b5 PMID: 15689740

37. Kao CL, Hsieh WL, Chern CM, Chen LK, Lin MH, Chan RC (2009) Clinical features of benign paroxysmal positional vertigo (BPPV) in Taiwan: differences between young and senior age groups. Arch Gerontol Geriatr 49(suppl.2)

38. Brosel S, Strupp M (2019) The vestibular system and ageing. In: Subcellular Biochemistry; 91. Springer, New York, pp 195-225

39. Sunami $K$, Tochino $R$, Tokuhara $Y$ (2005) Factors associated with recurrence of BPPV. Equilib. Res. 64:64-70. https://doi.org/10.3757/jser.64.64

40. Von Brevern M, Radtke A, Lezius F, Feldmann M, Ziese T, Lempert T, Neuhauser $\mathrm{H}$ (2007) Epidemiology of benign paroxysmal positional vertigo: a population based study. J Neurol Neurosurg Psychiatry 78(7):710-715

41. Kao C, Cheng Y, Leu H, Chen T, Ma H, Chen J, Lin S, Chan R (2014) Increased risk of ischemic stroke in patients with benign paroxysmal positional vertigo: a 9-year follow-up nationwide population study in Taiwan. Front Aging Neurosci 6:108. Published online. https://doi.org/10.3389/ fnagi.2014.00108

42. Bashir A, Lipton RB, Ashina S, Ashina M (2013) Migraine and structural changes in the brain: a systematic review and meta-analysis. Neurology 81(14):1260-1268. https://doi.org/10.1212/WNL.0b013e3182a6cb32 Epub 2013 Aug 28. PMID: 23986301; PMCID: PMC3795609

43. Zu Eulenburg P, Stoeter P, Dieterich M (2010) Voxel-based morphometry depicts central compensation after vestibular neuritis. Ann Neurol 68(2):241-249

44. Van der Holst HM, Tuladhar AM, Zerbi V, van Uden IWM, de Laat KF, van Leijsen EMC, de Leeuw FE (2018) White matter changes and gait decline in cerebral small vessel disease. Neurolmage Clin 17:731-738

45. Zheng JJ, Delbaere K, Close JC, Sachdev PS (2011) Lord SR (2011) Impact of white matter lesions on physical functioning and fall risk in older people: a systematic review. Stroke. 42(7):2086-2090. https://doi.org/10 1161/STROKEAHA.110.610360 Epub 2011 Jun 2. PMID: 21636821

46. Van Leijsen E, Van Uden I, Ghafoorian M, Bergkamp M, Lohner V, Kooijmans E, Van Der Holst H, Tuladhar A, Norris D, Van Dljk E, Rutten-Jacobs L, Platel B, Klijn C \& De Leeuw F. (2017) Nonlinear temporal dynamics of cerebral small vessel disease. Neurology 89(15):1569-1577

47. Wardlaw J, Chappell F, Valdés Hernández M, Makin S, Staals J, Shuler K, Thrippleton M, Armitage P, Munõz-Maniega S, Heye A, Sakka E, Dennis M
(2017) White matter hyperintensity reduction and outcomes after minor stroke. Neurology 89(10):1003-1010

\section{Publisher's Note}

Springer Nature remains neutral with regard to jurisdictional claims in published maps and institutional affiliations.

\section{Submit your manuscript to a SpringerOpen ${ }^{\circ}$ journal and benefit from:}

- Convenient online submission

- Rigorous peer review

- Open access: articles freely available online

- High visibility within the field

- Retaining the copyright to your article

Submit your next manuscript at $\boldsymbol{\nabla}$ springeropen.com 